03

\title{
Однонаправленные одноцикловые и субцикловые импульсы
}

\author{
(С) И.А. Со ${ }^{1, *}$, А.Б. Плаченов ${ }^{2, \dagger}$, А.П. Киселев ${ }^{3,4, \ddagger}$ \\ ${ }^{1}$ Невинпат, \\ 190000 Санкт-Петербург, Россия \\ ${ }^{2}$ МИРЭА - Российский технологический университет, \\ 119454 Москва, Россия \\ ${ }^{3}$ Санкт-Петербургское отделение Математического института им. В.А. Стеклова РАН, \\ 191023 Санкт-Петербург, Россия \\ ${ }^{3}$ Институт проблем машиноведения РАН, \\ 199178 Санкт-Петербург, Россия \\ *e-mail: irene_so@mail.ru \\ † e-mail: a_plachenov@mail.ru \\ ‡ e-mail: kiselev@pdmi.ras.ru
}

Поступила в редакцию 29.07.2020 г.

В окончательной редакции 29.07.2020 г.

Принята к публикации 14.08.2020 г.

\begin{abstract}
Представлено простое точное решение волнового уравнения в свободном пространстве. Оно описывает однонаправленные одноцикловые и субцикловые импульсы с конечной энергией. В зависимости от соотношения между входящими в решение свободными параметрами импульс может иметь блинообразный, шароподобный или иглообразный вид.
\end{abstract}

Ключевые слова: однонаправленные точные решения волнового уравнения, одноцикловые и субцикловые импульсы, блинообразные, шароподобные и иглообразные импульсы, фемтосекундная и аттосекундная оптика.

DOI: $10.21883 /$ OS.2020.12.50323.209-20

\section{1. Введение}

Развитие фемтосекундных и аттосекундных оптических технологий [1] делает актуальным математическое моделирование малоцикловых импульсов [2-4] без упрощающих предположений об их параксиальности. В настоящей заметке мы приводим простое точное осесимметрическое решение волнового уравнения

$$
\frac{\partial^{2} u}{\partial x^{2}}+\frac{\partial^{2} u}{\partial y^{2}}+\frac{\partial^{2} u}{\partial z^{2}}-\frac{1}{c^{2}} \frac{\partial^{2} u}{\partial t^{2}}=0
$$

$c=$ const $>0$ импульс однонаправленно распространяется вдоль оси $z$. Это решение обладает конечной энергией. В зависимости от выбора входящих в него произвольных параметров оно может описывать импульсы, имеющие в фокальной области блинообразную, шароподобную или иглообразную форму.

Наше решение существенно проще немногочисленных известных примеров однонаправленных импульсов $[3,4]$. Вывод его основан на разложении известного (не однонаправленного) сплэш-импульса (splash pulse) [5] на два слагаемых, удовлетворяющих уравнению (1) и описывающих однонаправленные импульсы, распространяющиеся в противоположные стороны. Математические вычисления, сами по себе элементарные, мы здесь опускаем.

\section{2. Однонаправленный импульс и его свойства}

Найденное нами решение имеет вид

$$
u=\frac{1}{S\left(z_{*}-S\right)},
$$

где обозначено

$$
S=\sqrt{c^{2} t_{*}^{2}-x^{2}-y^{2}},
$$

а звездочка означает комплексификацию:

$$
z_{*}=z+i \xi, \quad t_{*}=t+i \tau .
$$

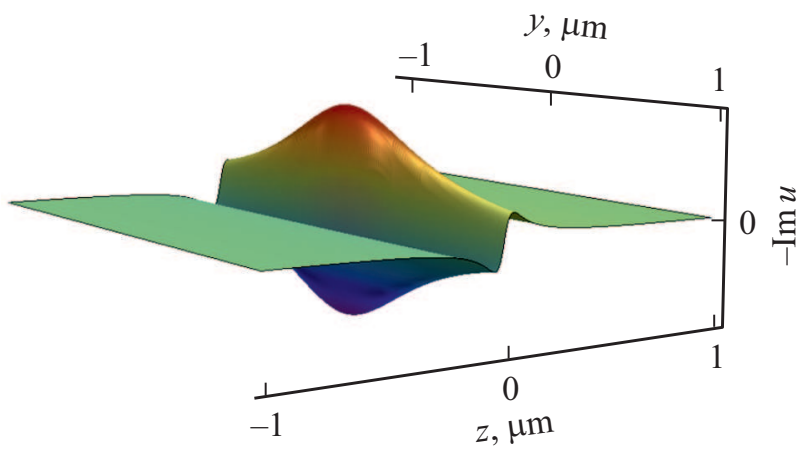

Рис. 1. Блинообразный импульс $-\operatorname{Im} u$ при $t=0, x=0$ $(\xi=16.66 \mu \mathrm{m}, c \tau=16.67 \mu \mathrm{m})$. 


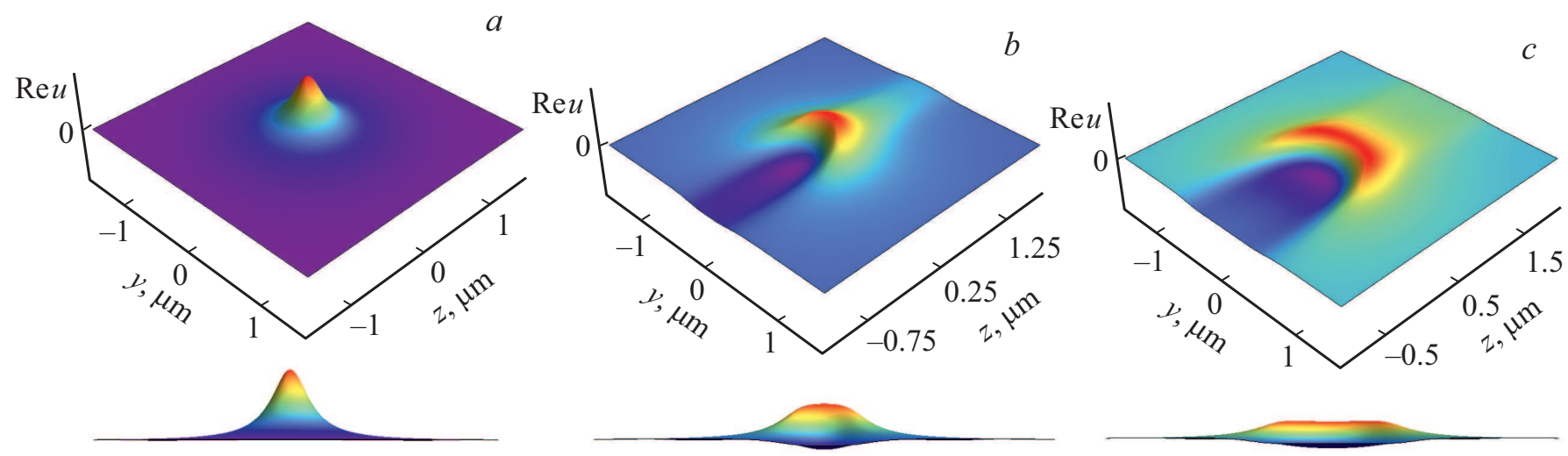

Рис. 2. Шароподобный импульс $\operatorname{Re} u$ при $t=0,0.25 / c, 0.5 / c \mu \mathrm{s}, x=0$ ( $\xi=0, c \tau=0.2 \mu \mathrm{m})$. Вид в перспективе и вид сзади.

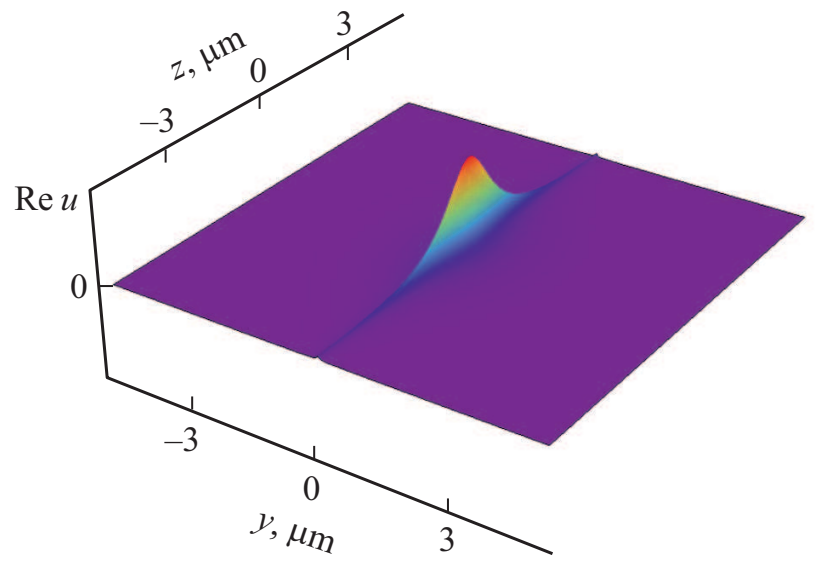

Рис. 3. Иглообразный импульс $\operatorname{Re} u$ при $t=0, x=0$ $(\xi=-0.975 \mu \mathrm{m}, c \tau=0.025 \mu \mathrm{m})$.

Здесь $\xi$ и $\tau \neq 0 \quad$ - свободные вещественные константы (ограничения, гарантирующие отсутствие сингулярностей, приведены ниже). Для определенности мы фиксируем ветвь корня так, что $\left.S\right|_{x=y=0}=c t_{*}$.

Легко проверяется, что при условии

$$
|\zeta|<c|\tau|
$$

функция (2) не имеет сингулярностей. Более тщательный анализ показывает, что область допустимых значений постоянной $\xi$ при данном $\tau$ шире. Именно, если $\tau>0$, то сингулярности у функции (2) отсутствуют при более слабом условии

$$
\xi<c \tau
$$

Соответственно для $\tau<0$ требуется неравенство $\xi>c \tau$. При выполнении этих условий на свободные постоянные можно показать, что функция (2) удовлетворяет волновому уравнению (1) при всех значениях координат $x, y$ и $z$ и времени $t$, а также имеет конечную энергию.
Решение (2) при больших отрицательных $t$ описывает волну, сходящуюся в полупространстве $z<0$ в фокальную область - окрестность начала координат. При $t \rightarrow+\infty$ волна расходится в полупространстве $z>0$. Фокусировка происходит при небольших значениях $t$.

При разных соотношениях между $\zeta$ и $\tau$ решение (2) описывает импульсы различного вида. Если $\xi$ близко к $c \tau$, то импульс в фокальной области блинообразный (см. рис. 1), при $|\xi| \ll c|\tau|$ - шароподобный (см. рис. 2), а если знаки $\xi$ и $\tau$ различны, причем $|\xi| \gg c|\tau|$, то форма импульса в фокальной области напоминает иглу (см. рис. 3). Мнимая часть функции (2) описывает симметричный биполярный импульс в фокусе. Вещественная часть соответствует субцикловому импульсу в фокусе. Субцикловые импульсы могут представлять интерес в приложениях, где желательно униполярное воздействие поля (см., например, [1,6]).

В дальнейшем мы собираемся показать, что рассмотренный нами импульс является представителем обширного класса однонаправленных решений волнового уравнения.

\section{Благодарности}

Авторы признательны С.А. Козлову и Н.Н. Розанову за стимулирующие обсуждения.

\section{Конфликт интересов}

Авторы заявляют, что у них нет конфликта интересов.

\section{Список литературы}

[1] Архипов Р.М., Архипов М.В., Шимко А.А., Пахомов А.В., Розанов Н.Н. // Письма в ЖЭТФ. 2019. Т. 110. В. 1. С. 9; Arkhipov R.M., Arkhipov M.V., Shimko A.A., Pakhomov A.V., Rosanov N.N. // JETP Letters. 2019. V. 110. N 1. P. 15.

[2] Feng S., Winful H.G., Hellwarth R.W. // Phys. Rev. E. 1999. V. 59. N 4. P. 4630. 
[3] Hernández-Figueroa H.E., Zamboni-Rached M., Recami E. Non-Diffracting Waves. John Wiley \& Sons, 2013. 509 p.

[4] Lekner J. Theory of electromagnetic pulses. Calif. Morgan \& Claypo, 2018. 100 p.

[5] Ziolkowski R.W. // J. Math. Phys. 1985. V. 26. N 4. P. 861.

[6] Архипов P.М., Архипов М.В., Розанов Н.Н. // Квант. электрон., 2020. T. 50. № 9. С. 801; Arkhipov R.M., Arkhipov M.V., Rosanov N.N. // Quantum Electron. 2020. V. 50. N 9, P. 801. 